Cahiers
de a $\begin{gathered}\text { Recherche } \\ \text { sur les Droits }\end{gathered}$

Cahiers de la recherche sur les droits

Fondamentaux fondamentaux

14 | 2016

Urbanisme et droits fondamentaux

\title{
Le droit de préemption et les exigences de la Convention européenne des droits de l'homme
}

The Right of Preemption and the Requirements of the European Convention on Human Rights

Jean-François Struillou

\section{OpenEdition}

Journals

Édition électronique

URL : https://journals.openedition.org/crdf/589

DOI : $10.4000 /$ crdf.589

ISSN : 2264-1246

Éditeur

Presses universitaires de Caen

Édition imprimée

Date de publication : 1 novembre 2016

Pagination : $59-71$

ISBN : 978-2-84133-838-2

ISSN : $1634-8842$

Référence électronique

Jean-François Struillou, «Le droit de préemption et les exigences de la Convention européenne des droits de l'homme », Cahiers de la recherche sur les droits fondamentaux [En ligne], 14 | 2016, mis en ligne le 01 octobre 2019, consulté le 14 novembre 2022. URL : http://journals.openedition.org/crdf/ 589 ; DOI : https://doi.org/10.4000/crdf.589 


\title{
Le droit de préemption et les exigences de la Convention européenne des droits de l'homme
}

\author{
Jean-François STRUILLOU \\ Directeur de recherche au CNRS \\ Droit et changement social (DCS, UMR 6297) \\ Faculté de droit et des sciences politiques de l'université de Nantes
}

I. Le principe du droit au respect des biens

A. Les exigences de la Cour européenne

B. Les incidences du principe en droit interne

1. Un apport souterrain

2. Des motifs d'interrogation

II. Le principe du droit à un procès équitable

A. La dimension incitative de la jurisprudence de la Cour

1. L'accès aux informations publiées au fichier immobilier

2. L'appréciation par le juge civil du caractère illégal des décisions de préemption

3. Les incidences de l'annulation de la décision de préemption sur la validité du transfert

B. Perspectives d'avenir

Tous ceux qui s'intéressent au droit de préemption sont aujourd'hui confrontés à un nouveau défi, celui de savoir si ce droit, qui en France a connu au cours de ces deux dernières décennies une extension sans précédent, est conforme aux principes issus de la Convention européenne des droits de l'homme (ci-après «la Convention $)^{1}$. Il importe donc de réexaminer à la lumière de ces principes les termes mêmes du compromis fixé par le droit national entre les prérogatives dont sont investies les collectivités territoriales et les droits des propriétaires, les instances de Strasbourg se montrant soucieuses d'un équilibre entre les seconds et les premières. Cette analyse paraît d'autant plus nécessaire que la majorité des propositions qui ont été formulées par le Conseil d'État en 1992 et en 2008 en vue d'inscrire le droit de préemption urbain dans ce juste équilibre ont, pour l'heure, été accueillies avec circonspection par le législateur, qui n'y a que très partiellement donné suite ${ }^{2}$. A été rejetée, par exemple, la plus originale des recommandations émises par le rapport de 2008 , laquelle suggérait - dans le but d'aboutir à une procédure plus équilibrée - de limiter les pouvoirs des collectivités territoriales en supprimant, dans certains cas, la possibilité pour ces dernières de recourir au juge de l'expropriation pour fixer le prix de l'immeuble ${ }^{3}$. Le

1. Voir F. Haumont, «La Cour européenne des droits de l'homme et le droit de préemption», L'actualité juridique. Droit administratif, 2008, p. 747; F. Bouyssou, «La préemption, l'urbanisme et le droit des gens», Revue de droit immobilier, 2015, p. 278.

2. Voir Conseil d'État, L'urbanisme: pour un droit plus efficace, Paris, La documentation française, 1992, p. 127 sq.; Conseil d'État, Le droit de préemption, Paris, La documentation française, 2008.

3. Sur ces propositions, voir J.-F. Struillou, «Le droit de préemption urbain à la croisée des chemins», Revue de droit immobilier, 2008 , p. 304. 
prix de la préemption aurait été ici celui figurant dans la déclaration d'intention d'aliéner.

Si la question de la conventionnalité du droit de préemption se pose avec acuité, c'est aussi parce qu'il est difficile pour ce dispositif d'échapper aux principes qui sont ceux du droit européen, dans la mesure où il existe désormais des procédures juridictionnelles de contrôle des lois qui assurent la conformité de celles-ci à la Convention européenne. Les justiciables français disposent depuis 1981, on le sait, d'un droit au recours individuel qui leur permet, après épuisement des voies de recours internes, de saisir directement la Cour européenne lorsque l'État n'a pas respecté les droits établis dans la Convention. La prééminence du droit de la Convention sur la loi est par conséquent bien moins théorique qu'autrefois, dès lors qu'il existe une juridiction pour juger les violations des droits de l'homme à l'échelle de l'Europe.

Cette configuration juridique nouvelle, qui témoigne d'une dilution de la souveraineté traditionnelle de la loi, est porteuse de dynamisme. Il s'agit là d'identifier les dispositions nationales susceptibles d'être affectées par la méta-norme, d'évaluer les risques contentieux consécutifs à cette situation nouvelle et, plus encore peut-être, d'élaborer des solutions de compromis qui, sans remettre en cause la primauté du droit européen, puissent apparaître comme étant de nature à ménager les traditions nationales ${ }^{4}$.

Les principes auxquels doit être confronté le droit interne sont au nombre de deux. Ils concernent d'une part le droit, substantiel, au respect des biens et, d'autre part, les garanties procédurales dont doivent, comme dans tout procès à caractère civil, disposer le propriétaire en cas de privation ou de limitation du droit au respect de ses biens.

L'article $1^{\text {er }} \mathrm{du}$ premier Protocole additionnel à la Convention (ci-après «l'article $\left.1^{\mathrm{er}} »\right)$ dispose en effet que:

Toute personne physique ou morale a droit au respect de ses biens. Nul ne peut être privé de sa propriété que pour cause d'utilité publique et dans les conditions prévues par la loi et les principes généraux du droit international.

Les dispositions précédentes ne portent pas atteinte au droit que possèdent les États de mettre en vigueur les lois qu'ils jugent nécessaires pour réglementer l'usage des biens conformément à l’intérêt général [...].

Quant à l'article 6, §1, de la Convention, il précise:

Toute personne a droit à ce que sa cause soit entendue équitablement, publiquement et dans un délai raisonnable, par un tribunal indépendant et impartial [...].

C'est à partir de ces deux textes qu'il convient de «revisiter» le droit français de la préemption.

\section{Le principe du droit au respect des biens}

Les termes employés par l'article $1^{\text {er }}$ pour assurer la protection du droit de chacun au respect de ses biens sont particulièrement lâches et flous, ces dispositions tendant avant tout à définir des principes directeurs qui renvoient à des choix éthiques ou à une normativité méta-juridique et non à fixer un statut précis et complet de la propriété privée immobilière. La Cour européenne s'est par conséquent astreinte à dégager les conditions auxquelles sont subordonnées les atteintes apportées à ce droit.

Il convient d'analyser cette jurisprudence (A) avant de rechercher si celle-ci est de nature à déstabiliser le droit interne (B).

\section{A. Les exigences de la Cour européenne}

Le droit pour chacun au respect de ses biens - lequel garantit en substance le droit de propriété - n'est pas, on le sait, un droit absolu. L'article $1^{\text {er }}$ n'exclut pas la possibilité pour les États d'y porter atteinte pour des motifs d'intérêt général, en particulier pour permettre à la puissance publique de procéder unilatéralement à l'acquisition de biens immobiliers indispensables à la réalisation des politiques d'aménagement, d'urbanisme ou d'environnement qu'elle entend engager. Face aux ingérences qui sont ainsi susceptibles d'affecter ce droit, la Cour européenne distingue traditionnellement trois «types» d'atteintes à partir du texte de la Convention ${ }^{5}$ : celles qui sont constitutives d'une privation de propriété au sens strict, celles qui procèdent d'une simple "réglementation» de l'usage des biens et celles qui, ne comportant ni dépossession, ni limitation de l'usage des biens, sont néanmoins attentatoires au principe du respect des biens.

S'agissant du droit de préemption, celui-ci s'analyse en principe comme une simple réglementation de l'usage des biens et relève par conséquent du deuxième alinéa de l'article $1^{\text {er }}$, lequel reconnaît aux États le droit de «mettre en vigueur les lois qu'ils jugent nécessaires pour réglementer l'usage des biens conformément à l'intérêt général » ${ }^{6}$. Le Conseil d'État paraît confirmer cette analyse en considérant que «toute décision de préemption d'un bien apporte une limitation au droit de propriété du vendeur et affecte à ce titre les intérêts de celui-ci» ${ }^{7}$. Cette atteinte est caractérisée, tout d'abord, par les limitations qu'apporte la préemption au droit du propriétaire de disposer librement de son bien. Ces limitations revêtent, en réalité, plusieurs aspects. Dans sa forme la moins préjudiciable, le propriétaire se voit, du fait de l'exercice du droit de préemption, imposer

4. Voir R. Hostiou, J.-F. Struillou, Expropriation et préemption: aménagement, urbanisme, environnement, $5^{\mathrm{e}}$ éd., Paris, Litec, 2016, p. 793.

5. Cour EDH, 23 septembre 1982, Sporrong et Lönnroth c. Suède, série A, no $52, \$ 61$.

6. Dans certaines circonstances - notamment lorsque l'atteinte au droit de propriété est considérée comme particulièrement importante - la Cour européenne peut aussi estimer que la mesure contestée entre dans le champ d'application de la règle fixée dans la première phrase de l'article $1^{\text {er }}$ du premier Protocole additionnel.

7. CE, 21 mai 2008, Commune de Houilles, Recueil Lebon, tables, p. 961. 
un partenaire contractuel autre que celui qu'il avait choisi. D’une manière plus contraignante, celui-ci peut également se voir imposer le prix de vente du bien. Ainsi, lorsque le titulaire du droit de préemption décide, avec l'accord du vendeur, de saisir le juge de l'expropriation, non seulement l'évaluation effectuée par ce dernier risque d'être inférieure au prix du marché, mais encore celle-ci a pour effet de lier le propriétaire quant au prix de revente de l'immeuble, cette procédure judiciaire ayant pour autre conséquence de retarder d'une manière non négligeable la réalisation de la vente. De façon plus drastique, l'exercice du droit de préemption provoque aussi parfois une modification radicale des conditions figurant dans le contrat de vente initial, notamment lorsque l'aliénation prévoit une contrepartie en nature. Dans cette hypothèse, qui recouvre notamment les échanges ou l'apport en société d'un immeuble, la contrepartie attendue par le propriétaire ne pourra pas être satisfaite et celui-ci devra se contenter, s'il accepte la vente, de l'allocation d'une somme d'argent, alors même qu'il avait initialement convenu d'une contrepartie autre. L'atteinte au droit de propriété du vendeur peut également être caractérisée par la privation d'une indemnisation raisonnablement en rapport avec la valeur des biens ou d'une espérance légitime d'une cession desdits biens à un prix plus favorable, en cas de préemption légale sans réalisation d'équipements publics sur la parcelle préemptée ${ }^{8}$.

L'acquéreur évincé devrait aussi pouvoir justifier, dans certaines circonstances, d'une atteinte au respect de ses biens au sens de l'article $1^{\text {er }}$, et ce alors même que la vente ne produirait ses pleins effets à son égard qu'après que le titulaire du droit de préemption a renoncé à exercer son droit. Dès lors qu'il ressort de la promesse de vente que la cession initialement envisagée est suffisamment probable, la Cour européenne pourrait ici considérer que «l'espérance légitime ${ }^{9}$ de l'acquéreur initial à devenir propriétaire du bien en cause - laquelle permet d'assimiler une «certitude de possession » à une "possession vraie ${ }^{10}-$ comme les droits patrimoniaux attachés à la promesse de vente doivent être qualifiés de «biens $»^{11}$.

Face aux atteintes qui peuvent être apportées de la sorte au droit de chacun au respect de ses biens, la Cour européenne paraît être en mesure d'assurer une protection effective de ce droit face aux prétentions de l'État, celle-ci n'ayant pas hésité à réécrire à cette fin les dispositions conventionnelles. Alors que la lettre de l'article $1^{\text {er }}$, alinéa 2, vise exclusivement à sauvegarder le «droit que possèdent les États» d'adopter certaines mesures affectant l'usage des biens - et non à protéger en tant que tel le droit de propriété qui serait affecté par ces mesures -, le juge européen a procédé à une tout autre lecture, beaucoup plus séduisante, de ces dispositions. Il y a également découvert une norme dont l'objet est de protéger les personnes contre les dispositions nationales relatives à la réglementation de l'usage des biens ${ }^{12}$.

Quant au degré de protection ainsi octroyé aux propriétaires, la faculté dont disposent les pouvoirs publics de réglementer l'usage des biens est subordonnée à trois conditions.

La première est d'ordre formel. Toute ingérence de l'autorité publique dans la jouissance du droit au respect des biens doit procéder de la loi, ou tout au moins reposer sur une loi. La portée de cette exigence ne saurait toutefois être surestimée, la Cour manifestant une très nette réticence à faire prévaloir, en cas de litige, sa propre appréciation sur celle des autorités nationales : c'est « aux autorités internes, notamment les cours et tribunaux", qu'il appartient, au premier chef, «d'interpréter et d'appliquer le droit interne et de se prononcer sur les questions de constitutionnalité ${ }^{13}$.

Le principe de légalité implique également l'existence de normes de droit interne suffisamment accessibles, précises et prévisibles ${ }^{14}$. Si cette condition est appliquée avec beaucoup de retenue, la Cour faisant preuve en règle générale d'une particulière mansuétude vis-à-vis du flou juridique dont sont affectées les limitations aux droits garantis par la Convention ${ }^{15}$, il faut relever que s'agissant du droit de préemption le manque de clarté de la loi a été observé dans les arrêts Beyeler c. Italie et Hentrich c. France ${ }^{16}$.

Dans la première affaire, était contesté l'exercice, par le ministère italien du Patrimoine culturel, du droit de préemption de l'État sur un tableau de Van Gogh dont le requérant avait fait l'acquisition. La Cour a ici constaté que la loi italienne manquait à certains égards d'intelligibilité, dans la mesure où elle prévoyait qu'en cas d'incomplétude de la déclaration d'intention d'aliéner un bien présentant

8. CE, 10 mars 2010, MM. Jean et Guy Lévy, Recueil Lebon, tables, p. 1014. Voir infra.

9. J. Fouchet, «L'espérance légitime dans le contentieux de l'urbanisme», Construction - Urbanisme, avril 2015, étude $\mathrm{n}^{\circ} 5$.

10. Voir Cour EDH, 29 novembre 1991, Pine Valley c. Irlande, n 12742/87; voir aussi CE, 10 mars 2010, MM. Jean et Guy Lévy: Bulletin de jurisprudence de droit de l'urbanisme, $\mathrm{n}^{\circ}$ 3, 2010, p. 215, concl. A. Courrèges.

11. Corinne Saint-Alary-Houin fait valoir que l'acquéreur évincé «est privé du bénéfice de son acquisition alors qu'il s'était mis d'accord avec le vendeur sur la chose et sur le prix. C’est bien de sa propriété qu'il est amputé» ("Approche conceptuelle du droit de préemption», La semaine juridique, édition notariale et immobilière, $\mathrm{n}^{\circ} 40,7$ octobre 2011, p. 1260).

12. Voir L. Condorelli, «Premier protocole additionnel. Article $1^{\mathrm{er}}$, in La Convention européenne des droits de l'homme. Commentaire article par article, $2^{\mathrm{e}}$ éd., L.-E. Pettiti, E. Decaux, P.-H. Imbert (dir.), Paris, Économica, 1999.

13. Cour EDH, 23 novembre 2000, Ex-roi de Grèce et autres $c$. Grèce, $\mathrm{n}^{\circ}$ 25701/94, $\$ 82$

14. Cour EDH, 22 septembre 1994, Hentrich c. France, série A, nº 296-A, p. 47, \$110; Cour EDH, 8 juillet 1986, Lithgow et autres c. Royaume-Uni, série $\mathrm{A}, \mathrm{n}^{\circ}$ 102, p. 47, $\$ 110$; Cour EDH, 5 janvier 2000, Beyeler c. Italie, $\mathrm{n}^{\circ} 33202 / 96$, p. 26, $\$ 109$.

15. S. Van Drooghenbroeck, La proportionnalité dans le droit de la Convention européenne des droits de l'homme. Prendre l'idée simple au sérieux, Bruxelles, Bruylant (Publications des Facultés universitaires Saint Louis), 2001, nº 97, p. 687.

16. Cour EDH, 5 janvier 200o, Beyeler c. Italie; Cour EDH, 22 septembre 1994, Hentrich c. France: Recueil Dalloz, 1995, jurispr., p. 465, note D. Fiorina ; Les petites affiches, $\mathrm{n}^{\circ}$ 69, 9 juin 1995, p. 8, note J.-F. Flauss. 
un intérêt culturel, le délai pour l'exercice du droit de préemption restait ouvert, la législation interne n'indiquant pas par quels moyens pareil vice pouvait être ultérieurement redressé. Cet élément d'incertitude dans la loi quant à la purge du droit de préemption n'a certes pas pour effet, à lui seul, d'amener à conclure que l'ingérence en cause était imprévisible ou arbitraire et par conséquent incompatible avec le principe de légalité. Force est néanmoins de constater que cet élément n'est pas pour autant dénué de conséquences juridiques: il entre «en ligne de compte dans l'examen de la conformité de la mesure litigieuse aux exigences du juste équilibre $»^{17}$.

Dans la seconde affaire, qui portait sur l'ancien droit de préemption exercé par l'administration fiscale pour insuffisance du prix payé par l'acquéreur d'un bien immobilier, la Cour a considéré que ce dispositif ne satisfaisait pas non plus aux exigences de précision et de prévisibilité qu'implique la notion de loi au sens de la Convention. Il est ici précisé avec force que le droit de préemption ne saurait être exercé de manière arbitraire, sélective, guère prévisible et de surcroît sans offrir les garanties procédurales élémentaires. Une décision de préemption ne peut ainsi avoir de légitimité en l'absence d'un débat contradictoire et respectueux du principe de l'égalité des armes, qui permette de discuter la question de la sousévaluation du prix et, par voie de conséquence, la position de l'Administration.

La deuxième exigence tient aux motifs de l'ingérence, la mesure en question devant être justifiée par l'intérêt général. Cette condition paraît pour l'heure faire l'objet d'un "non-contrôle». Il est certes exigé que, pour être conforme à la Convention, une réglementation de l'usage des biens doit être justifiée par l'intérêt général. Il n’en demeure pas moins que la Cour n'exerce aucun contrôle significatif sur les motifs qui ont inspiré l'exercice du droit de préemption, celle-ci se bornant à constater in abstracto que les dispositions contestées sont justifiées par des objectifs d'intérêt général. Cette réserve s'explique par la large marge d'appréciation qui est reconnue aux États, ceux-ci se trouvant en principe mieux placés que le juge international pour déterminer ce qui est d'intérêt général, dès lors qu'ils ont une connaissance directe de leur société et de ses besoins ${ }^{18}$. Cette marge d'appréciation est d'autant plus ample que la notion d'intérêt général n'a jusqu'ici fait l'objet d'aucune définition et que la Cour hésite à s'immiscer dans l'examen de questions politiques, économiques et sociales qu'implique la détermination de l'intérêt général. Aussi est-il impensable que le juge européen puisse faire autre chose que de s'incliner devant la conception de l'intérêt général adoptée par chaque État, sauf cas de fourvoiement ou de déraison manifeste. Une telle situation est susceptible de se produire lorsque le droit de préemption a été exercé en vue de faire obstacle à un acquéreur jugé indésirable en raison de ses origines, ou en vue de mettre en échec la construction d'un lieu de culte et lorsque ces détournements de pouvoir n'ont pas été sanctionnés par le juge national du fait des difficultés rencontrées par les requérants pour établir la preuve de cette illégalité.

Quant à la troisième exigence, elle est relative à la proportionnalité de l'atteinte portée au droit de chacun au respect de ses biens, celle-ci devant ménager «un juste équilibre entre les exigences de l'intérêt général de la communauté et les impératifs de la sauvegarde des droits fondamentaux de l'individu ${ }^{19}$. La Cour s'attache en conséquence à vérifier si les victimes de cette ingérence n'ont pas eu à supporter à cette occasion une charge "disproportionnée et excessive». Si cette construction prétorienne présente indubitablement l'avantage de donner aux juges de Strasbourg un cadre rationnel à l'exercice de leur pouvoir contentieux, la portée de ce contrôle ne doit pas pour autant être surestimée ${ }^{20}$. Plusieurs facteurs sont à l'origine de ce constat.

Tout d'abord, ce contrôle dit du « juste équilibre » est un contrôle de proportionnalité au sens étroit, dans la mesure où sa mise en œuvre se borne à mettre en balance les exigences de l'intérêt général et les effets de la réglementation sur le droit de propriété du requérant. Le juge ne vérifie pas le caractère idoine de la mesure contestée, ni l'existence d'alternatives moins restrictives à la mesure contestée - comme le recours à l'acquisition amiable -, ces appréciations relevant du pouvoir d'appréciation de l'État pour décider des limitations au droit de propriété. Tout au plus l'absence de nécessité de la mesure peut-elle être un élément - parmi d'autres - à prendre en considération.

La portée de ce contrôle paraît ensuite d'autant plus réduite que la Cour européenne reconnaît aux États une très grande marge d'appréciation pour décider des limitations qu'il convient d'apporter au droit de propriété. Plusieurs facteurs sont à l'origine de cette situation. Le premier tient aux termes mêmes de l'article $1^{\text {er }}$ qui précisent explicitement que c'est aux États qu'il revient d'adopter les lois qu'ils jugent nécessaires pour réglementer l'usage des biens ${ }^{21}$. Le deuxième ressort de la nature même du droit auquel il est porté atteinte. Dès lors que le droit de propriété jouit a priori d'une protection faible par rapport à d'autres droits garantis par la Convention et que les sociétés démocratiques connaissent toutes des restrictions à ce droit, la Cour tend à reconnaître aux États une marge de manœuvre plus grande. Quant au troisième,

17. Cour EDH, 5 janvier 2000, Beyeler c. Italie, p. $27, \$ 110$.

18. Cour EDH, 21 février 1986, James et autres c. Royaume-Uni, série A, nº 98, p. 32, \$46; Cour EDH, 5 janvier 20oo, Beyeler c. Italie.

19. Cour EDH, 23 septembre 1982, Sporrong et Lönnroth c. Suède, $\$ 69$.

20. Voir J.-F. Struillou, «La conformité du droit français de l'urbanisme avec l'article $1^{\text {er }}$ du Premier protocole additionnel à la Convention européenne des droits de l'homme», in D'urbanisme et d'environnement. Liber Amicorum Francis Haumont, C.-H. Born, F. Jongen (dir.), Bruxelles, Bruylant, 2015, p. 349.

21. Voir supra. 
il est étroitement lié au but poursuivi par les restrictions apportées à l'exercice du droit de propriété. L'intérêt général occupant une place prééminente dans le cadre des politiques d'aménagement du territoire et de protection de l'environnement, la Cour considère par suite que les États doivent disposer d'une marge d'appréciation plus grande que lorsque sont en jeu des droits exclusivement civils $^{22}$. Cette importante marge d'appréciation a de la sorte pour effet d'affaiblir le contrôle exercé, la Cour considérant que le juste équilibre est rompu uniquement «si la personne a subi une charge spéciale et exorbitante», c'est-à-dire une charge disproportionnée au regard de la finalité d'intérêt général poursuivie par les pouvoirs publics.

Reste à préciser quels sont les critères pris en compte jusqu'à présent pour apprécier si la mise en œuvre du droit de préemption ménage ce «juste équilibre».

La Cour de Strasbourg manifeste ici une vigilance toute particulière quant aux conditions d'exercice du droit de préemption, s'agissant des motifs susceptibles de le justifier et des conditions de dédommagement des victimes de la préemption. Entrent également en compte la possibilité pour le requérant de contester la mesure de préemption prise à son égard, ainsi que le comportement des parties au litige, y compris les moyens employés par l'État et leur mise en œuvre. Dans l'arrêt Hentrich c. France (précité), il a par exemple été jugé que le «juste équilibre» avait été rompu parce que l'État était autorisé à faire usage de son droit sans avoir à indiquer les motifs, en droit et en fait, de sa décision et parce que l'indemnité versée par l'Administration était de nature à léser l'acquéreur du bien préempté, sans que celui-ci ait la possibilité de contester le montant de cette indemnisation. De même, dans l'arrêt Beyeler c. Italie, la Cour a estimé que l'exigence $\mathrm{du}$ «juste équilibre» avait été méconnue dès lors que les conditions dans lesquelles le droit de préemption avait été exercé avaient permis à l'État d'acquérir le tableau litigieux à un prix sensiblement inférieur à sa valeur marchande. Sont ainsi condamnées les "manouvres» autour de l'exercice du droit de préemption, l'État ayant tiré un enrichissement injuste de l'incertitude qui a régné pendant plusieurs années sur l'exercice ou non de cette prérogative.

Ces exigences sont-elles de nature à remettre en cause le droit français de la préemption?

\section{B. Les incidences du principe en droit interne}

Alors que les exigences manifestées par la Cour européenne devraient amener à reconsidérer certaines conditions de mise en œuvre du droit de préemption, le principe du droit de chacun au respect de ses biens n'a eu pour l'heure qu'un impact limité sur le droit interne.

\section{Un apport souterrain}

À titre préventif, on peut avancer que le «droit au respect des biens » a été à l'origine de quelques innovations législatives et d'une solution jurisprudentielle destinée à rendre le droit français compatible avec les exigences manifestées par la Cour de Strasbourg.

En premier lieu, le souci de respecter la Convention a amené les autorités nationales à modifier la durée de validité de la zone d'aménagement différé (ZAD), c'està-dire la période de temps pendant laquelle le titulaire du droit de préemption peut exercer son droit au sein de cette zone.

Pour limiter dans le temps les sujétions qui pèsent sur les propriétaires et aussi afin d'éviter que les collectivités publiques ne reportent indéfiniment la réalisation de l'opération d'aménagement prévue dans cette zone, tout en leur laissant le temps nécessaire pour faire entrer dans leur patrimoine les immeubles qui leur sont indispensables, le législateur a limité la durée de validité de l'acte instituant la ZAD - qui était de quatorze ans - à «six ans renouvelables». Cette évolution, qui est issue de l'article 6, I, de la loi no 2010-597 du 3 juin 2010 relative au Grand Paris, marque une rupture, pour autant que jusqu'ici les interventions du Parlement en la matière s'étaient toujours soldées par un allongement de la durée de validité des ZAD. Selon les travaux parlementaires, cette évolution vise avant tout à prendre davantage en considération les exigences issues de l'article $1^{\mathrm{er}}$.

En deuxième lieu, la Convention européenne a également joué un certain rôle dans la modification des dispositions qui régissent la «date de référence», qui est la date à laquelle se situe le juge de l'expropriation pour qualifier le bien de terrain à bâtir ou, pour les autres biens, pour déterminer l'usage effectif qui en était fait à cette date. Ces règles visent à prémunir les collectivités contre les risques de spéculation foncière artificielle liée à l'annonce par l'acte créant la ZAD d'une opération d'intérêt général à venir, en «gelant» la valeur du bien préempté à la date de la création ou du renouvellement de la $\mathrm{ZAD}$.

Les dispositions qui fixent désormais la date de référence à la date du dernier renouvellement de l'acte créant la ZAD - et non plus à la date de sa création - semblent plus favorables aux propriétaires. Elles visent à faire en sorte que la date de référence ne remonte pas trop loin dans le temps et, ainsi, elles tendent à supprimer le risque de qualifier certains biens situés en ZAD de terrains agricoles ou naturels, alors même que ces derniers ont été aménagés ou construits. Comme le souligne le rapport du Sénat sur le projet de loi relatif au Grand Paris, l'approche consistant à fixer une date de référence trop éloignée « serait contraire au droit de propriété consacré par l'article $1^{\mathrm{er}} \mathrm{du} 1^{\mathrm{er}}$ protocole additionnel à la Convention européenne ${ }^{23}$. Reste que ces règles présentent toujours un certain intérêt pour

22. Voir Cour EDH, 29 mars 2010, Brosset-Triboulet et autres c. France, nº 34078/02.

23. J.-P. Fourcade, Rapport fait au nom de la commission spéciale sur le projet de loi relatif au Grand Paris, n 366 , enregistré à la présidence du Sénat le 25 mars 2010, en ligne: https://www.senat.fr/rap/lo9-366/log-3661.pdf, p. 74. 
l'Administration tout en désavantageant le propriétaire: elles font obstacle à ce qu'une éventuelle modification ou révision d'un document de planification urbaine, intervenue après le renouvellement de la $\mathrm{ZAD}$, ne vienne augmenter le coût de l'opération.

En troisième lieu, on peut relever un arrêt du Conseil d'État qui tend également à rendre notre droit compatible avec les exigences manifestées par la Cour européenne.

Pour bien comprendre la portée et l'intérêt de cette décision, rappelons que le «droit de rétrocession » qui est accordé à l'ancien propriétaire ou à l'acquéreur évincé au cas où le titulaire du droit de préemption déciderait de ne pas affecter l'immeuble à un projet d'intérêt général s'apparente davantage à une "fausse fenêtre juridique» qu'à une véritable garantie, qui permettrait effectivement à ces derniers de s'assurer que l'immeuble préempté a bien reçu une destination d'intérêt général. Si le titulaire décide d'utiliser ou d'aliéner à d'autres fins un bien acquis depuis moins de cinq ans, il doit simplement informer de sa décision l'ancien propriétaire - voire l'acquéreur évincé et leur proposer l'acquisition de ce bien en priorité. Passé ce délai de cinq ans, le titulaire est délié de toute obligation à l'égard des parties à la vente initialement convenue, et il peut par conséquent revendre ou conserver en toute liberté le bien au cas, entre autres, où le projet en vue duquel la préemption a été utilisée est abandonné.

La non-conventionnalité de ce dispositif a été évoquée à plusieurs reprises, dans la mesure où la Cour européenne paraît exiger, pour qu'un «juste équilibre» soit assuré entre les exigences de l'intérêt général et la sauvegarde du droit de chacun au respect de ses biens, que les immeubles acquis par la mise en œuvre de prérogatives de puissance publique soient affectés à une destination d'intérêt général et qu'en l'absence d'affectation les anciens propriétaires bénéficient d'un droit de rétrocession ou, tout au moins, soient indemnisés. Au cas où une collectivité locale a procédé à la réalisation, par voie d'expropriation, de réserves foncières en vue de la réalisation d'un habitat social et où il apparaît, plus de quinze ans après cette acquisition, que cet objectif n'a pas été rempli, la Cour européenne a ainsi considéré, à défaut de rétrocession, que les expropriés, indûment privés de la plus-value acquise par ces terrains, ont été victimes d'une violation de l'article $1^{\text {er } 24}$.

La confrontation accrue du droit interne aux exigences de la Convention européenne a conduit le Conseil d'État à transposer l'esprit de cette jurisprudence dans le domaine de la préemption ${ }^{25}$. Le juge administratif paraît admettre désormais qu'en l'absence de toute réalisation d'équipements publics sur le bien légalement préempté et en cas, par conséquent, de rétention dudit bien pendant une longue période sans justification d'utilité publique, la décision de préemption fait aussi peser sur l'ancien propriétaire une charge disproportionnée de nature à caractériser une méconnaissance des stipulations de l'article $1^{\text {er }}$. Dans cette situation, l'ancien propriétaire, qui peut subir une atteinte excessive à son droit de propriété, doit donc pouvoir engager la responsabilité du titulaire du droit de préemption, et ce alors même que l'exercice du droit de préemption est légal. Cette atteinte pourrait justifier la réparation du préjudice correspondant à la différence entre le montant du prix offert par l'acquéreur évincé et la valeur du bien préempté, si cette dernière est inférieure. En revanche, l'ancien propriétaire ne serait pas autorisé à réclamer la plus-value engendrée par le bien au cours de la période pendant laquelle le bien n'a pas été affecté à une destination d'intérêt général car, comme l'a fort bien démontré Anne Courrèges, pendant cette période le propriétaire doit être regardé comme s'étant, de toute façon, déjà défait de sa propriété au profit de l'acquéreur évincé ${ }^{26}$.

\section{Des motifs d'interrogation}

Il n'est pas certain que les évolutions ainsi constatées soient suffisantes pour mettre la France à l'abri d'une condamnation par les instances de Strasbourg. Des motifs d'interrogation subsistent sur au moins trois points.

Le premier tient au fait que l'exercice du droit de préemption emporte parfois des conséquences suffisamment sérieuses pour que la mesure atteigne un seuil de gravité certain et fasse peser sur le propriétaire une "charge disproportionnée». Il en va ainsi lorsque la collectivité renonce à exercer son droit alors que le juge de l'expropriation a fixé le prix de l'immeuble. Cette renonciation, qui par la force des choses risque d'intervenir alors que la déclaration d'intention d'aliéner a été adressée depuis déjà très longtemps, est susceptible d'entraîner différents préjudices pour le propriétaire ${ }^{27}$. D'une part, l'utilisation du droit de préemption est de nature à «dévaluer» le bien ou, tout au moins, à constituer un élément plutôt négatif aux yeux de futurs acheteurs. D'autre part, le vendeur se trouve dans l'impossibilité de disposer du prix figurant dans la promesse de vente entre la date de cession prévue par cet acte et la date à laquelle il retrouvera un autre acquéreur. Enfin, le propriétaire peut subir un autre préjudice financier lorsqu'après la renonciation de la collectivité, il est contraint de vendre son bien à un prix moindre que celui fixé dans la promesse de vente initiale. Les règles de la préemption sont ici d'autant

24. Cour EDH, 2 juillet 2002, Motais de Narbonne c. France, no 48161/99: L'actualité juridique. Droit administratif, 2002, p. 1226, note R. Hostiou, Cahiers du CREDHO, n 9, 2003, p. 207, note J.-P. Demouveaux.

25. CE, 10 mars 2010, MM. Jean et Guy Lévy: L'actualité juridique. Droit administratif, 2010, p. 1098, note R. Hostiou; Bulletin de jurisprudence de droit de l'urbanisme, $\mathrm{n}^{\circ}$ 3, 2010, p. 215, concl. A. Courrèges; L'actualité juridique. Droit immobilier, 2011, p. 70, note F. Bouyssou et C. Teisseyre.

26. «La perte de plus-value potentielle ne concerne pas directement l'ancien propriétaire, qui n'en aurait de toute façon pas eu la jouissance puisqu'il aurait vendu son bien" (CE, 10 mars 2010, MM. Jean et Guy Lévy: Bulletin de jurisprudence de droit de l'urbanisme, $\mathrm{n}^{\circ}$ 3, 2010, p. 215, concl. A. Courrèges).

27. CA Montpellier, 19 mai 2015, EPFL Perpignan-Méditerranée c. M. Areny, n 13/oo121, JurisData, n² 2015-012284. En l'espèce la procédure de préemption a duré près de quatre ans, la Cour d'appel ayant été saisie par le titulaire du droit de préemption. 
plus « déséquilibrées » que le propriétaire ne peut vendre son bien au prix initialement convenu - sauf à souscrire une nouvelle déclaration d'intention d'aliéner -, celui-ci étant tenu par le prix fixé par le juge de l'expropriation, et ce pendant un délai de cinq ans. Les mêmes préjudices peuvent être subis par le propriétaire, lorsque le titulaire du droit de préemption renonce à la préemption au cours de la procédure, sans que pour autant le juge de l'expropriation n'ait été saisi.

Aujourd'hui, ces dommages ne donnent pas lieu à réparation lorsque la décision de préemption - à laquelle il est finalement renoncé - n'est pas entachée d'illégalité. La préemption étant réputée légale, la demande d'indemnisation du vendeur ne peut être rattachée au cas classique de la responsabilité pour faute à raison d'une préemption illégale ${ }^{28}$.

Cet état du droit n'est pas sans susciter certaines réserves. La mise en œuvre du contrôle de proportionnalité pourrait amener la Cour européenne à considérer - dans une situation particulière - qu'à la suite d'une renonciation à une préemption légale, le vendeur a été privé, en raison de l'échec de la vente initiale, de l'espérance légitime d'une cession de son bien à un prix plus favorable et qu'ainsi la décision de renonciation à la préemption légale a fait peser sur lui une «charge disproportionnée» de nature à caractériser une méconnaissance de l'article $1^{\mathrm{er}}$.

L'autre motif d'interrogation porte sur les dispositions qui soumettent au droit de préemption urbain et au droit de préemption dans les ZAD, les cessions de la majorité des parts d'une société civile immobilière (SCI), ainsi que "les cessions conduisant un acquéreur à détenir la majorité des parts de ladite société ${ }^{29}$. S’il s'agit là d'adapter la préemption à la réalité des transactions immobilières, en particulier lorsque celles-ci se déclinent sur le mode du droit incorporel à travers la cession de parts de sociétés, ces bouleversements ne sont pas sans soulever un certain nombre de problèmes, en particulier au regard de l'article $1^{\mathrm{er}}$.

Le premier tient aux difficultés que pourrait rencontrer la collectivité devenue associée pour se faire attribuer l'immeuble. Comme il l'a été souligné,
[...] le transfert de propriété d'une part entraîne uniquement le transfert des prérogatives politiques - droit de vote principalement - et financières - vocation à partager le bénéfice et à contribuer aux pertes notamment - attachées à cette part, mais en aucun cas d'un quelconque droit direct sur un immeuble ressortissant du patrimoine de la sociétézo.

Il est par conséquent difficile - voire impossible - pour le titulaire du droit de préemption d'affecter le terrain propriété de la SCI au projet qui a justifié l'exercice du droit de préemption, lequel vise une action ou une opération d'aménagement. Il en va ainsi tout particulièrement lorsque le droit de préemption est exercé sur « les cessions conduisant un acquéreur à détenir la majorité des parts de ladite société», ces cessions risquant de conduire «à des situations quelque peu surprenantes si l'on admet que le DPU [droit de préemption urbain] permettra ainsi à son titulaire d'intégrer une SCI pour quelques parts seulement $»^{31}$.

Ce premier problème en soulève un deuxième. Dès lors que le bien ne peut que difficilement être affecté à une destination d'intérêt général, se pose la question de la conformité de ces règles à l'article $1^{\mathrm{er}}$. Les biens ayant été acquis en recourant à une technique juridique qui apporte une limitation au droit de propriété du vendeur, il paraît nécessaire, pour qu'un «juste équilibre» soit ménagé entre les exigences de l'intérêt général de la communauté et les impératifs de la sauvegarde du droit au respect des biens, que la collectivité donne au bien la destination d'intérêt général prévue par la décision de préemption. Dans le domaine de l'expropriation, la Cour européenne a ainsi jugé que l'effectivité de l'affectation du bien exproprié conditionne la régularité de l'opération ${ }^{32}$. Dès lors que par leur nature même les biens en cause ne peuvent en pareil cas être utilisés à des fins d'intérêt général et que l'ancien propriétaire ne bénéficie d'aucun droit de rétrocession - ce dernier n'étant applicable qu'aux «terrains» acquis par exercice du droit de préemption ${ }^{33}-$, l'extension du champ de la préemption aux parts de SCI semble en décalage par rapport aux exigences manifestées par les instances de Strasbourg. Quoi qu'il en soit, et

28. CAA Marseille, 9 mai 2014, Torres c. commune de Marseille, $\mathrm{n}^{\circ} 12 \mathrm{MA02067}$ : Bulletin de jurisprudence de droit de l'urbanisme, $\mathrm{n}^{\circ}$ 6, 2014, p. 501, chron. M. Revert. Voir aussi CA Paris, 28 janvier 2016, Communauté d'agglomération Plaine-commune c. $M^{m e}$ Assogba, n $14 / 19637$, JurisData, $\mathrm{n}^{\circ}$ 2016-001623. Notons toutefois qu'en matière d'expropriation le refus de poursuivre le processus amorcé et de mener à son terme la procédure d'expropriation, alors même qu'il n'est pas constitutif d'une faute, est susceptible d'engager la responsabilité de l'Administration sur la base du principe d'égalité face aux charges publiques, sous la simple condition de l'anormalité et de la spécialité du préjudice consécutif à cette situation. CE, 23 décembre 1970, EDF c. Farsat, Recueil Lebon, p. 790 : L'actualité juridique. Droit administratif, 1971, p. 96, concl. M. Kahn; CE, 3 mars 1976, Girouard, Recueil Lebon, p. 118: La semaine juridique, 1977, II, 18698, note A. Homont. Voir aussi R. Hostiou, J.-F. Struillou, Expropriation et préemption..., p. 615.

29. Ces dispositions, qui sont codifiées à l'article L. 213-1 $3^{\circ}$ du Code de l'urbanisme, sont issues de la loi no $2014-366$ du 24 mars 2014 pour l'accès au logement et un urbanisme rénové (loi ALUR). Voir J.-F. Struillou, «L'impact de la loi ALUR sur le régime du droit de préemption», Revue française de droit administratif, 2014, p. 576; J.-P. Meng, "Loi pour l'accès au logement et un urbanisme rénové», Bulletin du Cridon de Paris, $\mathrm{n}^{\mathrm{o}} 6-7,15$ mars-1 $^{\mathrm{er}}$ avril 2014, p. 50.

30. W. Altide, D. Dutrieux, "Alur", parts de SCI et droit de préemption urbain», La semaine juridique, édition notariale et immobilière, n 42 , 17 octobre 2014, $\mathrm{n}^{\circ}$ 1302. Voir aussi J.-P. Meng, «Les nouvelles règles du droit de préemption compliquent-elles réellement les ventes?», Revue de droit immobilier, 2015, p. 228.

31. G. Durand-Pasquier, «Florilège de quelques difficultés soulevées par les droits de préemption et autres priorités d’achat nouvellement institués ou modifiés», Construction - Urbanisme, avril 2015, focus n³ 30. Voir W. Altide, D. Dutrieux, «"Alur”, parts de SCI...».

32. Cour EDH, 2 juillet 2002, Motais de Narbonne c. France.

33. Selon les termes mêmes de l'article L. 215-22 du Code de l'urbanisme. 
contrairement aux apparences qui laisseraient à penser que le droit de préemption est tout aussi bien adapté aux parts sociales qu'aux immeubles, il semble, au contraire, que le problème soulevé soit ici plus complexe, ne seraitce que parce que la préemption de la majorité des parts d'une société ne résout pas la question de l'appropriation de l'immeuble, qui est - faut-il le rappeler? - la "cause juridique» de la décision de préemption. Plutôt que de vouloir étendre sans cesse ces dispositions, peut-être serait-il par conséquent plus opportun de les abroger.

Enfin, les exigences issues de l'article $1^{\text {er }}$ méritent une attention particulière, s'agissant du "droit de rétrocession" de l'ancien propriétaire et de l'acquéreur évincé, les conditions dans lesquelles ce droit peut être mis en œuvre n'étant pas - on l'a dit - de nature à permettre à ces derniers de contrôler l'affectation des biens préemptés. Dès lors qu'il devient plus difficile de contester utilement devant les juridictions administratives, par le biais du contrôle des motifs, la légalité d'une décision de préemption, la question se pose de savoir s'il ne convient pas ici, pour répondre aux exigences européennes, d'instaurer un contrôle plus vigilant sur le «suivi» de l'immeuble préempté. Ce dernier ayant été acquis par le biais de prérogatives de puissance publique qui apportent une limitation au droit de propriété du vendeur et affectent à ce titre les intérêts de celui-ci ${ }^{34}$, il paraît nécessaire, pour qu'un juste équilibre soit ménagé «entre les exigences de l'intérêt général de la communauté et les impératifs de la sauvegarde des droits fondamentaux de l'individu», que la collectivité publique soit tenue de donner au bien une destination conforme à l'intérêt général et que l'ancien propriétaire - voire l'acquéreur évincé - bénéficie d'un droit de rétrocession effectif au cas où la collectivité n'aurait pas satisfait aux obligations qui lui incombent ${ }^{35}$. Il a ainsi été proposé «de renforcer et de rendre plus effectif» le mécanisme actuel en conférant à l'acquéreur évincé et à l'ancien propriétaire une nouvelle faculté, celle de faire constater que le bien préempté n'a pas reçu ou a cessé de recevoir au bout de cinq ans à compter de son acquisition une destination d'intérêt général et de réclamer pour ce motif la «rétrocession» de l'immeuble ${ }^{36}$.

\section{Le principe du droit à un procès équitable}

L'article 6, \$1, de la Convention européenne - qui reconnaît le droit pour toute personne à ce que sa cause soit entendue équitablement, publiquement, et dans un délai raisonnable, par un tribunal indépendant et impartial - a eu une incidence plus forte sur le droit français de la préemption: il est à la source d'évolutions jurisprudentielles récentes destinées à rendre le droit interne "euro-compatible».

\section{A. La dimension incitative de la jurisprudence de la Cour}

Les mécanismes européens de protection des droits de l'homme ont avant tout une fonction préventive, les autorités nationales étant invitées à anticiper et à faire pénétrer en droit interne les principes issus de la Convention. Les exemples qui suivent, dans lesquels sont impliqués la Cour de cassation, le Conseil d'État et le Tribunal des conflits, rendent compte de la volonté des juridictions nationales de prendre acte des exigences européennes, de faire pénétrer celles-ci en droit interne et de prémunir de la sorte le droit français de la préemption des risques contentieux consécutifs à une éventuelle saisine des instances de Strasbourg.

\section{L'accès aux informations publiées au fichier immobilier}

Il s'agit d'abord du droit des propriétaires d'accéder aux informations publiées au fichier immobilier, en cas de fixation judiciaire du prix de l'immeuble préempté par le juge de l'expropriation. Le principe de l'égalité des armes suppose ici «un juste équilibre entre les parties» au procès, chacune de celles-ci devant se voir offrir une possibilité «raisonnable» de présenter sa cause dans des conditions qui ne la placent pas dans une situation de «net désavantage » par rapport à son ou à ses adversaires. Le titulaire du droit de préemption comme le commissaire du gouvernement ne sauraient par conséquent bénéficier d'un accès privilégié au fichier immobilier contenant les informations "pertinentes» sur l'état du marché foncier, l'accès à ces sources - qui est de nature à permettre au propriétaire de l'immeuble objet de la décision de préemption d'apprécier le bien-fondé du montant du prix proposé par la collectivité - devant être égal pour toutes les parties concernées.

On sait que le rôle prépondérant que jouait le commissaire du gouvernement tout au long de la procédure judiciaire en fixation de l'indemnité d'expropriation et les facilités qui lui étaient offertes en matière d'accès à ces informations concernant les mutations de comparaison et la différence de traitement qui en résultait entre expropriant et exproprié ont été stigmatisés par la Cour européenne. Dans un arrêt célèbre du 24 avril 2003, Yvon c. France, les instances de Strasbourg ont considéré que

34. CE, 21 mai 2008, Commune de Houilles.

35. J.-F. Struillou, «Les transformations de la question foncière. Droit de préemption et liberté contractuelle», Études foncières, n 100 , novembredécembre 2002, p. 43. Sur les limites du droit de rétrocession pour compenser l'atteinte à l'intérêt général causé par l'irrégularité d'une préemption sans projet, voir J. Morand-Deviller, «Le droit de préemption: transparence et cohérence», Bulletin de jurisprudence de droit de l'urbanisme, $\mathrm{n}^{\circ} 6,2006$, p. 402.

36. Conseil d'État, Le droit de préemption, p. 70 
cette situation créait au détriment des expropriés «un déséquilibre incompatible avec le principe de l'égalité des armes » et que la procédure judiciaire se trouvait de la sorte en contradiction avec les exigences issues de l'article $6, \$ 1$, relativement à un procès équitable ${ }^{37}$. Il en est résulté l'adoption d'un décret dont l'objectif ouvertement proclamé était de procéder au «toilettage» du Code de l'expropriation aux fins que celui-ci prenne effectivement en compte les exigences européennes ${ }^{38}$. Après que plusieurs voix ont souligné le caractère insuffisant de ces modifications - dans la mesure où les agents du Domaine bénéficiaient toujours auprès du service de la conservation des hypothèques de facilités d'accès dont les expropriés ne bénéficiaient pas -, les pouvoirs publics ont tenu à compléter les dispositions en cause. La loi $\mathrm{n}^{\circ}$ 2006-872 du 13 juillet 2006 a ainsi procédé à une modification de l'article $135 \mathrm{~B}$ du Livre des procédures fiscales, ces dispositions imposant désormais à l'administration fiscale de transmettre gratuitement, à leur demande, aux propriétaires faisant l'objet d'une procédure d'expropriation les éléments qu'elle détient au sujet des valeurs foncières déclarées à l'occasion des mutations intervenues dans les cinq dernières années. Les propriétaires de biens sur lesquels un droit de préemption est exercé bénéficient des mêmes droits ${ }^{39}$.

C'est dans ce contexte qu'a été soulevée devant la Cour de cassation la question de savoir quelles sont les valeurs foncières déclarées à l'occasion des mutations intervenues dans les cinq dernières années que l'Administration est tenue d'adresser au propriétaire dont le bien a été préempté. Le propriétaire s'étant heurté à un refus de l'ancienne Conservation des hypothèques de lui transmettre ces informations, au motif que ne pouvaient lui être communiquées que les informations concernant des parcelles «identifiées", la Cour de cassation a dans un arrêt en date du 7 novembre 2012 - rendu au visa de l'article 6, \$1 - sanctionné fermement ce comportement de l'Administration consistant à restreindre l'accès au fichier immobilier ${ }^{40}$. Selon le juge de cassation, les exigences manifestées par la Cour européenne quant au principe de l'égalité des armes impliquent ici que le droit pour les propriétaires des biens préemptés de recevoir du service de la publicité foncière les éléments d'information qu'elle détient au sujet des valeurs foncières ne soit pas restreint, sauf pour l'État à encourir le risque d'aboutir à une situation qui pourrait être perçue par la Cour européenne comme une rupture de l'égalité des armes.

Il s'agit là pour la Cour de cassation moins de corriger les anomalies du droit de préemption que de protéger les droits qui sont conférés au propriétaire d'un bien qui a fait l'objet d'une décision de préemption, ces droits découlant explicitement des exigences de l'article $6, \$ 1$, de la Convention européenne. On peut aussi voir dans cette décision la confirmation explicite du poids des exigences nouvelles, désormais incontournables, issues de la Convention européenne.

\section{L'appréciation par le juge civil du caractère illégal des décisions de préemption}

Les règles qui gouvernent l'exercice du droit de préemption présentent cette particularité qu'elles relèvent, pour partie, du droit public et, pour partie, du droit privé. Le contentieux de la préemption est, en conséquence, partagé entre chacun des deux ordres de juridiction, avec tous les risques de dysfonctionnement qu'implique une pareille situation. Aussi bien la mise en œuvre de cette technique que son contrôle génère un contentieux qui se partage entre juridictions administratives - la décision de préemption étant une décision administrative -, juridictions civiles - le contrat conclu entre la collectivité publique et le propriétaire s'analysant comme un contrat de droit privé $^{41}$ - et juge de l'expropriation enfin, seul habilité, en cas de désaccord, à déterminer la valeur du bien.

Parmi les difficultés consécutives à cette mixité, le problème de l'appréciation par le juge civil de la légalité de la décision de préemption occupe une place de choix. Il n'est pas exceptionnel que la résolution par le juge judiciaire d'un litige concernant le contrat de vente conclu entre le propriétaire et le titulaire du droit de préemption implique d'apprécier la légalité de la décision par laquelle le droit de préemption est exercé. La question s'est ainsi posée de savoir si le juge judiciaire est ici compétent, ou bien si, dans le cadre d'une question préjudicielle, il doit en renvoyer la connaissance à la juridiction administrative. Si, en vertu du principe de séparation des autorités administratives et judiciaires, la juridiction administrative est seule compétente pour connaître des recours tendant à l'annulation ou à la réformation de cet acte, mais aussi pour statuer, le cas échéant par voie de question préjudicielle, sur toute contestation de sa légalité, soulevée à l'occasion d'un litige relevant à titre principal de l'autorité judiciaire, cette répartition des compétences constitue pour le justiciable une source de complication, d'allongement des procédures et de renchérissement de leur coût. La complexité et la lourdeur de ce système présentent en outre l'inconvénient de faciliter, voire de favoriser, les comportements dilatoires,

37. Cour EDH, 24 avril 2003, Yvon c. France, n 44962/98, p. 21, \$37: L'actualité juridique. Droit immobilier, 2003, p. 361 , note D. Musso; Recueil Dalloz, 2003, p. 2456, note R. Hostiou; La semaine juridique, édition administrations et collectivités territoriales, n 22, 26 mai 2003 , p. 716, note R. Noguellou; Cahiers du CREDHO, nº 10, 2004, p. 93, note F. Rolin; L'actualité juridique. Droit administratif, 2004, p. 1441, tribune R. Hostiou; L'actualité juridique. Droit administratif, 2003, p. 1924, chron. J.-F. Flauss; Revue de droit de l'urbanisme, 2003, p. 425, étude J.-F. Struillou.

38. Voir R. Hostiou, «Une tentative de "conventionnalisation" du Code de l'expropriation", L'actualité juridique. Droit administratif, 2005, p. 1382; D. Musso, «Une réforme en trompe-l'œil», L'actualité juridique. Droit immobilier, 2005, p. 543

39. C'est ce qui résulte de l'article L. 213-4 du Code de l'urbanisme.

40. Cass., $3^{\mathrm{e}}$ civ., 7 novembre 2012, SARL Iliac c. Communauté d'agglomération creilloise, $\mathrm{n}^{\circ}$ 11-17.803, Bulletin civil III, $\mathrm{n}^{\circ} 166:$ L'actualité juridique. Droit immobilier, 2013, p. 53, note R. Hostiou; Revue trimestrielle de droit immobilier, no 1, 2013, note J.-F. Struillou; Recueil Dalloz, 2012 , p. 2663 JurisData, n' 2012-025052; Droit de l'aménagement, de l'urbanisme et de l'habitat, n 17, 2013, p. 355, chron. J.-F. Struillou.

41. TC, 9 décembre 1996, $M^{m e}$ Duhamel, $\mathrm{n}^{\circ}$ 96-02.994: Bulletin de jurisprudence de droit de l'urbanisme, $\mathrm{n}^{\circ}$ 3, 1997, p. 197, concl. R. Abraham. 
le titulaire du droit de préemption pouvant être tenté de jouer avec les difficultés pour le requérant d'identifier le «bon interlocuteur», susceptible de lui apporter une réponse adéquate en temps utile.

Une décision récente du Tribunal des conflits visant à «aménager» la répartition des compétences entre les deux ordres de juridiction et, par là même, à tempérer les inconvénients liés au dualisme juridictionnel n'est pas étrangère à l'objectif de prendre davantage en compte les exigences issues de la Convention européenne ${ }^{42}$.

La compétence du juge civil pour constater l'illégalité de la décision par laquelle une personne publique renonce à exercer son droit de préemption et pour en écarter l'application a ainsi été admise lorsqu'il résulte d'une «jurisprudence établie» que cet acte est entaché d'illégalité. Il y a là une application remarquable d'une jurisprudence importante du Tribunal des conflits, laquelle autorise les tribunaux judiciaires à déclarer, à l'occasion d'un litige relevant à titre principal du juge judiciaire, l'illégalité des actes administratifs manifestement irréguliers ${ }^{43}$. Il n'y a donc plus lieu désormais de faire le détour par le juge administratif lorsqu'il ressort clairement de la jurisprudence du Conseil d'État que l'acte administratif contesté est manifestement illégal: le juge civil de l'action est dans ce cas habilité à apprécier lui-même cette illégalité.

Cette solution est fondée sur la volonté d'éviter que l'exigence touchant au respect des délais raisonnables, composante du droit à un procès équitable garanti par l'article $6, \$ 1$, ne vienne perturber le contentieux de la préemption. Ce danger a été très bien mis en lumière par le commissaire du gouvernement dans ses conclusions. Après avoir observé que huit années après la décision initiale de préemption, cinq voies de recours ont été exercées par le titulaire de ce droit, dont quatre à ce jour sans succès, M. Girard souligne à juste titre que:

[...] le débat ouvert sur la renonciation à préemption de la propriété prête le flanc à une critique évidente sur le caractère excessif de la durée de la procédure qui pourrait se retourner, à terme, contre la communauté d'agglomération et les deux ordres juridictionnels actionnés ${ }^{44}$.

Sont ainsi dénoncées, sans que ne soit remis en cause le dualisme juridictionnel dans son principe même, les conséquences de celui-ci en tant que facteur d'allongement du règlement du litige, l'accumulation des lenteurs consécutives à l'obligation faite au requérant de saisir successivement le juge judiciaire et le juge administratif étant susceptible d'être analysée comme une violation du droit du requérant à voir sa cause entendue dans un délai raisonnable. Ce risque paraît d'autant moins théorique que dans un domaine proche, celui de l'expropriation pour cause d'utilité publique, la Cour européenne des droits de l'homme n'a pas hésité, à partir du constat de la durée excessive d'une procédure contentieuse intentée devant les deux ordres de juridiction, à sanctionner les dysfonctionnements consécutifs à l'intervention des juges administratif et judiciaire en ce domaine ${ }^{45}$.

Relevons encore que la façon dont le Tribunal des conflits a ici redessiné le partage des compétences entre les deux ordres de juridiction est diamétralement opposée à celle qui avait été proposée par le Conseil d'État. Dans son rapport sur le droit de préemption, celui-ci suggérait «d'unifier au sein de la juridiction administrative le contentieux des décisions de préemption et des contrats connexes à ces décisions » aux fins de pallier les difficultés qu'engendre en la matière un double contentieux. Si cette proposition de redécoupage des compétences au profit du juge administratif apparaît comme étant fort éloignée de la solution consacrée par le Tribunal des conflits - laquelle vise, au contraire, à renforcer fort opportunément pour les justiciables les pouvoirs du juge judiciaire ${ }^{46}$-, ces deux approches obéissent néanmoins à une même logique. Elles montrent que le temps des renvois préjudiciels systématiques est dépassé. Le souci d'une bonne administration de la justice - en tout point conforme aux principes du droit au procès équitable - conduit de plus en plus à habiliter le juge de l'action à se faire, à certaines conditions, juge de l'exception, quitte à assouplir les règles régissant le partage des compétences entre les deux ordres de juridiction et, plus particulièrement, celles qui réservent aux juridictions administratives une compétence exclusive pour apprécier la légalité des décisions administratives. Plutôt que de craindre cette évolution qui, selon certains, pourrait conduire les juridictions judiciaires à qualifier de "manifeste» une illégalité qui ne l'est pas, dans le but d'éviter un sursis à statuer et le renvoi d'une question préjudicielle au juge administratif, il faut, selon nous, au contraire s'en féliciter et se fier finalement à l'aptitude du juge judiciaire à discerner s'il y a dans la question soulevée une difficulté réelle, de nature à faire naître un doute dans un esprit éclairé.

\section{Les incidences de l'annulation de la décision de préemption sur la validité du transfert}

L'hétérogénéité des règles de droit applicables au droit de préemption et la complexité du contentieux qui en

42. TC, 16 juin 2014, $M^{m e}$ Semavoine c. Communauté d'agglomération de La Rochelle, $\mathrm{n}^{\circ} \mathrm{C}_{3953}$, Recueil Lebon, tables, p. 584 : L'actualité juridique. Droit administratif, 2014, p. 1658, note J.-F. Struillou; Revue de droit immobilier, 2014, p. 660, obs. M. Revert; Revue de jurisprudence de droit des affaires, $\mathrm{n}^{\circ} 4,2015, \mathrm{n}^{\circ} 257$; JurisData, $\mathrm{n}^{\circ}$ 2014-013620; La semaine juridique, édition administrations et collectivités territoriales, $\mathrm{n}^{\circ} 26,30$ juin 2014 , act. 529, F. Tesson; Droit de l'aménagement, de l'urbanisme, de l'habitat, 19 éd., GRIDAUH (dir.), Paris, Le moniteur, 2015, p. 394, chron. J.-F. Struillou.

43. TC, 17 octobre 2011, SCEA du Chéneau, no C3828-3829: Revue française de droit administratif, 2011, p. 1122, concl. J.-D. Sarcelet; p. 1129, note B. Seiller; p. 1136, note A. Roblot-Troiziet; L'actualité juridique. Droit administratif, 2012, p. 27, chron. M. Guyomar et X. Domino; Droit administratif, janvier 2012, comm. n 10 , note F. Melleray.

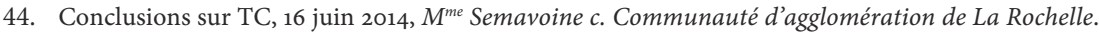

45. Cour EDH, 21 février 1997, Guillemin c. France, Recueil des arrêts et décisions de la Cour EDH, 1996, I, p. 149: L'actualité juridique. Droit administratif, 1997, p. 399, note R. Hostiou.

46. Voir supra. 
résulte soulèvent un autre problème majeur. Il s'agit de la question de savoir quelles sont les conséquences à tirer de l'annulation de la décision de préemption par le juge administratif, lorsque cette censure intervient plusieurs mois, voire plusieurs années après la conclusion de la vente issue du processus de préemption, c'est-à-dire à un moment où la propriété de l'immeuble a déjà été transférée à la collectivité ${ }^{47}$. On sait que l'annulation pour excès de pouvoir est ici de nature à susciter des difficultés d'exécution particulière chaque fois que les acquisitions réalisées par voie de préemption ont conduit la collectivité à revendre l'immeuble, ou encore à réaliser sur cet immeuble des travaux dont la démolition est toujours difficilement envisageable, que ce soit pour des raisons d'ordre juridique, technique, économique, financier ou social ${ }^{48}$.

Confrontés à ces difficultés cardinales, le Conseil d'État et le législateur ont apporté certains aménagements au droit interne, dans le but de prévenir le risque de « discordances» entre les décisions rendues par les deux ordres de juridiction, de faire obstacle à ce qu'une situation de fait soit entérinée au bénéfice de l'Administration, ou encore d'éviter l'allongement de la durée du règlement du litige. Ces aménagements relèvent des principes que la Cour européenne a mis en œuvre dans l'arrêt Guillemin c. France, l'idée étant ici de faire en sorte que les garanties inhérentes au contentieux de la préemption puissent être considérées comme suffisantes au regard des exigences de l'article $6, \S 1$, et de l'article $1^{\text {er }}$.

Le premier de ces aménagements vise le référésuspension, lequel présente un intérêt certain dans le contentieux de la préemption pour autant que, dans ce domaine, il est essentiel d'empêcher qu'une décision de préemption de légalité douteuse ne crée une situation de droit ou de fait sur laquelle il sera difficile de revenir en cas d'annulation ultérieure de l'acte contesté. Les conditions de recevabilité et d'obtention du référé-suspension, tout comme les effets de la suspension, ont ainsi été interprétées par le Conseil d'État de telle manière que l'obtention du référé soit facilitée et que cette mesure d'urgence, lorsqu'elle est ordonnée par le juge adminis- tratif, soit de nature, selon les cas, à faire obstacle à la prise de possession du bien par la collectivité ou, si le transfert de propriété a déjà été opéré à la date à laquelle il statue, à empêcher cette collectivité de faire usage de certaines des prérogatives qui s'attachent au droit de propriété, de manière à éviter que l'usage ou la disposition qu'elle fera de ce bien jusqu'à ce qu'il soit statué sur le litige au fond, ne rende irréversible la décision de préemption, sous réserve cependant qu'à cette date la collectivité n'en a pas déjà disposé, par exemple par la revente du bien à un tiers ${ }^{49}$. Le décalage du transfert de propriété à la date la plus tardive des dates auxquelles seront intervenus le paiement et l'acte authentique - institué par la loi ALUR du 24 mars 2014 - devrait également donner au juge administratif, en cas de demande de référé-suspension, un temps précieux pour lui permettre, si les conditions posées à l'article L. 521-1 du Code de justice administrative sont réunies, de paralyser tous les effets de la décision de préemption ${ }^{50}$. Le risque que la collectivité publique ne cède le bien à un tiers avant même que le juge de l'urgence ne se soit prononcé en dernier ressort - auquel cas la demande de suspension devient sans objet, la décision étant considérée comme entièrement exécutée - semble ainsi moins important que par le passés1.

Quant à la seconde mesure, elle a consisté à définir «les conséquences de droit» de l'annulation de la décision de préemption par le juge administratif, après que le transfert de propriété a eu lieu. Le titulaire du droit de préemption doit ici proposer à l'ancien propriétaire l'acquisition du bien en priorité, et ce dès que l'acte litigieux est annulé ou déclaré illégal par la juridiction administrative ${ }^{52}$. Si ce dernier renonce à racheter l'immeuble ${ }^{53}$, le titulaire devra proposer l'acquisition à l'acquéreur évincé, à la condition toutefois que le nom de ce dernier ait figuré dans la déclaration d'intention d'aliéner, ce qui a pour inconvénient de limiter de manière draconienne les droits de l'acquéreur évincé au cas où, pour faire obstacle à une préemption en considération de la personne, celui-ci aurait refusé que son nom soit mentionné dans cet acte.

Ce dispositif - issu de la loi ALUR du 24 mars 2014 - a pour effet d'inverser la solution qui avait été retenue par

47. Voir J.-F. Struillou, «Remarques sur l'efficacité des annulations pour excès de pouvoir dans le contentieux de la préemption», in Mélanges en l'honneur de Henri Jacquot, F. Priet, E. Fâtome, Y. Jégouzo, J.-P. Lebreton, G. Marcou, M. Pertué, F. Priet (dir.), Orléans, Presses universitaires d'Orléans, 2006, p. 545.

48. On rencontre les mêmes difficultés dans le contentieux de l'expropriation. Voir R. Hostiou, La «Cour européenne des droits de l'homme et la théorie de l'expropriation indirecte", Revue trimestrielle des droits de l'homme, n 70, avril 2007, p. 385 ; R. Hostiou, «Requiem pour une théorie défunte que d'aucuns estiment pouvoir ressusciter", in Confluences: mélanges en l'honneur de Jacqueline Morand-Deviller, M. Deguergue, L. Fonbaustier (dir.), Paris, Montchrestien, 2007, p. 579; R. Noguellou, «L'expropriation indirecte», Droit administratif, avril 2007, p. 3.

49. CE, 13 novembre 2002, M. Hourdin, Recueil Lebon, p. 396 : Revue du droit immobilier, 2003, p. 205, chron. P. Soler-Couteaux; Bulletin de jurisprudence de droit de l'urbanisme, $\mathrm{n}^{\circ}$ 6, 2002, p. 460, concl. J.-H. Stahl; Droit de l'aménagement, de l'urbanisme et de l'habitat, $\mathrm{n}^{\circ} 7,2003, \mathrm{p} .341$, chron. J.-F. Struillou. CE, 23 juillet 2003, Société Atlantique Terrains, Recueil Lebon, tables, p. 915 et p. 925 : Bulletin de jurisprudence de droit de l'urbanisme, n 6, 2003, p. 422, concl. P. Fombeur, p. 430, obs. J.-C. Bonichot; Defrénois, 2009, p. 671, note J.-P. Meng; Droit de l'aménagement, de l'urbanisme et de l'habitat, $\mathrm{n}^{\circ}$ 8, 2004, p. 545, chron. J.-F. Struillou. Sur le détail de cette jurisprudence et ses implications, voir R. Hostiou, J.-F. Struillou, Expropriation et préemption..., p. 636 sq.

50. Voir Conseil d'État, Le droit de préemption, p. 70.

51. CE, sect., 23 juin 2006, Société Actilor: L'actualité juridique. Droit immobilier, 2007, p. 150, note J.-F. Struillou.

52. Art. L. 213-11-1 du Code de l'urbanisme.

53. Il est réputé avoir renoncé à l'acquisition à défaut d'acceptation dans le délai de trois mois à compter de la notification de la décision juridictionnelle devenue définitive. 
le Conseil d'État dans l'arrêt Bour ${ }^{54}$, dans la mesure où il appartenait auparavant à l'Administration de proposer à l'acquéreur évincé puis, le cas échéant, au propriétaire initial, d'acquérir le bien. Ce changement d'angle - inspiré selon les débats parlementaires par le souci de prendre acte des exigences manifestées par la Cour européenne s'explique par le souci d'éviter une situation inextricable dans laquelle le titulaire du droit de préemption proposerait à l'acquéreur évincé le bien et conclurait avec lui un nouveau contrat, alors que l'ancien propriétaire souhaiterait quant à lui recouvrer la propriété de son ancien immeuble. Ce dernier aurait alors la possibilité de saisir le juge judiciaire afin qu'il constate la nullité du contrat de droit privé conclu sur la base d'une décision illégale, avec le risque, au cas où le juge judiciaire ferait droit à cette requête, d'aboutir à une solution différente de celle ordonnée par le juge administratif. Pour pallier ces risques de contrariété liés à l'intervention des deux ordres de juridiction dans un même contentieux, le Conseil d'État avait recommandé une solution plus radicale - qui n'a pas été retenue - consistant à « unifier au sein de la juridiction administrative le contentieux des décisions de préemption et des contrats directement connexes à ces décisions ", et à «donner au juge administratif la possibilité de prononcer la nullité de ces contrats en conséquence de l'annulation de la décision de préemption ${ }^{55}$.

Quels que soient les mérites de cette action conjointe des juridictions et du législateur, on est cependant tenté de douter du caractère suffisant de ces réformes.

\section{B. Perspectives d'avenir}

On soulignera, tout d'abord, s'agissant du contentieux relatif aux conséquences de l'annulation de la décision de préemption, que la répartition des compétences en la matière reste difficilement intelligible. C'est au juge administratif qu'il incombe d'ordonner au titulaire du droit de préemption de proposer à l'ancien propriétaire ou, à défaut, à l'acquéreur évincé l'acquisition du bien illégalement préempté. C'est en revanche au juge de l'expropriation qu'il revient, en l'absence d'accord amiable, de fixer le prix de l'immeuble «rétrocédé». Enfin, il n'est pas exclu - malgré la réforme issue de la loi ALUR du 24 mars 2014 - que le juge de droit commun soit saisi par l'une des parties, afin qu'il constate la nullité du contrat conclu sur la base d'une décision de préemption illégale ${ }^{56}$. La complexité de ce système ainsi que sa lourdeur, tout comme le caractère non suspensif des recours, présentent toujours un risque, celui d'engendrer des dysfonctionnements et des retards dans la résolution des litiges. Un dossier récent témoigne ainsi de l'enlisement de la procédure juridictionnelle par laquelle l'acquéreur évincé suite à une décision de préemption illégale demandait au juge judiciaire d'annuler la vente intervenue entre la commune et le vendeur. Au bout de onze ans de procédure devant le juge judiciaire, celui-ci a finalement «botté en touche» - provisoirement - et invité les requérants à saisir le juge administratif aux fins que celui-ci apprécie l'impact de l'annulation de la décision de préemption sur la vente conclue à l'issue de l'exercice de cette prérogative ${ }^{57}$.

En outre, les obstacles juridiques à la cession des terrains illégalement préemptés au propriétaire ou à l'acquéreur évincé n'ont pas disparu. D'une part, le juge administratif ne peut être tenu d'enjoindre à l'Administration de céder le bien illégalement préempté à l'acquéreur évincé ou au vendeur lorsque le rétablissement de la situation initiale - c'est-à-dire le retour du bien dans le patrimoine du vendeur ou de l'acquéreur évincé - est de nature à porter une atteinte «excessive » à l'intérêt général. D'autre part, lorsque le bien litigieux a été revendu, ni les dispositions de l'article L. 911-1 du Code de justice administrative, ni aucune autre disposition n'autorise la juridiction administrative, saisie en vue de faire exécuter l'annulation de la seule décision de préemption, à prescrire des mesures qui tendraient à la remise en cause de la revente du bien ${ }^{58}$. Cet état du droit, qui fait obstacle à ce que les victimes de la préemption soient placées dans une situation équivalente à celle où ils se trouveraient s'il n'y avait pas eu excès de pouvoir, oblige à relativiser les évolutions récentes.

Reste un dernier point sur lequel l'attention des autorités nationales mérite indubitablement d'être attirée. Il s'agit des difficultés qu'est susceptible de susciter une utilisation abusive du droit de préemption, le législateur ayant refusé d'y apporter de nouveaux éléments de réponse. Plusieurs rapports ont pourtant révélé que ce dernier était parfois détourné de son objet et exercé à des fins discriminatoires - consistant, par exemple, à «faire barrage à un acquéreur indésirable, en raison de son identité ou de ses origines ${ }^{59}$ - mais aussi que ces détournements condamnables restaient difficiles à sanctionner, dès lors que la jurisprudence de la chambre criminelle de la Cour

54. CE, sect., 26 février 2003, M. et $M^{m e}$ Bour, Recueil Lebon, p. 59 : Bulletin de jurisprudence de droit de l'urbanisme, $\mathrm{n}^{\circ} 2,2003$, p. 106, concl. P. Fombeur; p. 121, obs. J.-C. Bonichot; Les petites affiches, n' 164,18 août 2003, p. 4, chron. F. Melleray; Defrénois, 2003, p. 1336, note J.-P. Meng; Revue de droit immobilier, 2003, p. 377 et 379, obs. P. Soler-Couteaux; Droit de l'aménagement, de l'urbanisme et de l'habitat, n 8, 2004, p. 549, chron. J.-F. Struillou; Bulletin juridique des collectivités locales, $\mathrm{n}^{\circ} 10,2003, \mathrm{p} .710$, note E. Carpentier.

55. Conseil d'État, Le droit de préemption, p. 71

56. Cette compétence «concurrente» pour déterminer les conséquences de l'annulation de l'acte détachable sur le contrat repose, d'une part, sur le pouvoir d'injonction dont dispose désormais le juge administratif pour assurer l'exécution de ses décisions et, d'autre part, sur la compétence du juge judiciaire pour constater la nullité d'un contrat de vente de droit privé qui a été conclu sur la base d'un acte administratif irrégulier.

57. CA Versailles, 24 novembre 2011, M. Doublet c. commune d'Hermanville-sur-Mer, $\mathrm{n}^{\mathrm{o}}$ 10/05380, JurisData, $\mathrm{n}^{\circ} 2011-030664$.

58. CE, sect., 26 février 2003, M. et $M^{m e}$ Bour.

59. Conseil d'État, Le droit de préemption, p. 29 sq. 
de cassation interprète de manière rigoureuse les dispositions de l'article 432-7 du Code pénal, qui réprime le délit de discrimination par personne exerçant une fonction publique $^{60}$. Si un amendement proposant de compléter ces dispositions aux fins que ces déviances - de nature à affecter la légitimité de cet instrument - puissent être effectivement sanctionnées a bien été déposé au cours des débats parlementaires sur le projet de loi ALUR, celui-ci a néanmoins été rejeté aux motifs que les règles proposées étaient de nature à susciter
[...] chez les élus de très fortes craintes au moment même où [le Parlement] cherch[e] à sécuriser l'exercice du droit de préemption afin de permettre une meilleure mobilisation du foncier ${ }^{61}$.

C’est dire que malgré les améliorations législatives apportées, la loi ALUR n'a pas clos le débat sur les mesures à adopter pour aboutir à une procédure de préemption plus équilibrée, plus respectueuse des droits de l'homme et conforme aux exigences manifestées par la Cour européenne.

60. Cass. crim., 21 juin 2011, n 10-85.641, Bulletin criminel, $\mathrm{n}^{\circ}$ 143: Dalloz actualité, 7 juillet 2011, obs. M. Bombled; Droit pénal, octobre 2011, comm. M. Véron; Revue de droit immobilier, 2011, p. 507, obs. G. Roujou de Boubée. Voir aussi J. Lasserre Capdeville, «Le risque de discrimination dans le cadre de l'exercice du droit de préemption", L'actualité juridique. Collectivités territoriales, 2013, p. 235.

61. C. Bérit-Débat, in Sénat, Compte rendu intégral, séance du 26 octobre 2013, p. 10465. 